Sección dos: Textos

Educación y Exclusión social

\title{
De la exclusión social al reconocimiento de la dignidad en los contextos educativos ${ }^{1}$
}

From social exclusion to the recognition of dignity in educational contexts

Edward Amorocho Herrera

eduar333@hotmail.com

Alonso Malpica Capacho

alonsokapacho@hotmail.com

\section{Resumen}

El presente artículo tiene por finalidad, establecer algunos aspectos fundamentales del reconocimiento en los escenarios educativos, puesto que esto responde a todo tipo de exclusión social que se presentan en los contextos escolares. Al respecto, se abordó cómo se contextualiza el tema de la exclusión a nivel social y educativo. Luego, se desarrolló la cohesión que existe en la dignidad humana y el reconocimiento, puesto que esto contribuye a legitimar prácticas de reconocimiento en los procesos educativos. Posteriormente, se abordó la configuración de la alteridad como posibilidad de encuentro y de reconocimiento en el contexto de la educación, desde una perspectiva transformadora en los procesos dialógicos que se consolidan en las prácticas del aula. Para finalizar se encontró la importancia de incentivar una pedagogía del reconocimiento en las prácticas educativas, a partir del aprendizaje del servicio en las instituciones educativas.

Palabras clave: Educación, exclusión social, reconocimiento, dignidad y aprendizaje cooperativo.

\begin{abstract}
The purpose of this article is to establish some fundamental aspects of recognition in educational settings, since this responds to all types of social exclusion that arise in school contexts. In this regard, it was addressed how the issue of social and educational exclusion is contextualized. Then, the cohesion that exists in human dignity and recognition was
\end{abstract}

${ }^{1}$ Recibido: 30/04/2019 Evaluado: 02/05/2019 Aceptado: 05/05/2019 
developed, since this helps to legitimize recognition practices in educational processes. Subsequently, the configuration of alterity as a possibility of encounter and recognition in the context of education was approached from a transformative perspective in the dialogical processes that are consolidated in the classroom practices. Finally, it was found important to encourage a pedagogy of recognition in educational practices, based on the learning of the service in educational institutions

Keywords: Educatión, social exclusion, recognition, dignity and cooperative learning

\section{Introducción}

El presente artículo tiene por finalidad, establecer algunos aspectos fundamentales del reconocimiento en los escenarios educativos, puesto que esto responde a todo tipo de exclusión social que se presentan en los contextos escolares. En un primer momento, se abordará cómo se contextualiza el tema de la exclusión a nivel social y educativo, desde la problemática que conlleva hablar de exclusión social y cómo repercute en la educación.

En un segundo momento, se desarrollará la cohesión que existe en la dignidad humana y el reconocimiento, dado que esto contribuye a legitimar prácticas de reconocimiento en los procesos educativos donde se reconocen las libertades individuales y sociales de los sujetos.

En un tercer momento, se abordará la configuración de la alteridad como posibilidad de encuentro y de reconocimiento en el contexto de la educación, desde una perspectiva transformadora en los procesos dialógicos que se consolidan en las prácticas del aula. Para finalizar se dilucidará la importancia de promover una pedagogía del reconocimiento en las prácticas educativas, a partir del aprendizaje del servicio el cual constituye la capacidad del estudiante de incentivar en su proceso de aprendizaje habilidades de cooperación, de comunicación y de empatía en la comunidad educativa en beneficio de la equidad social.

\section{Idea 1: El marco de la exclusión social}

La noción de exclusión social ha adquirido un creciente protagonismo en nuestra sociedad como consecuencia de las transformaciones de las principales dimensiones tradicionales de organización económica y sociocultural en el contexto actual. Es así como el auge del capitalismo exacerbado así como de la globalización han generado que en muchos contextos sociales se desarrollen exclusiones sociales que afectan como tal el desarrollo humano de las comunidades. A su vez, el desarrollo emergente de un nuevo modelo de sociedad también ha sido analizado por Tezanos quien constata la existencia de un cambio en esas categorías propias de sociedades industriales -considerando "el sistema económico, el sistema social y el sistema político-, cambio que ha propiciado la configuración, entre otras muchas denominaciones, de una «sociedad tecnológica" (Jimenez, M., Luego, J., y Tabernet. N., 2009, p.11).

La exclusión social expresa la nueva configuración de las desigualdades dentro de la actual «sociedad tecnológica», de la información y del conocimiento donde el "saber constituye una 
parte más grande que nunca del valor de numerosos productos y servicios" (p12). En este nuevo contexto social, la exclusión no sólo implica la reproducción de las desigualdades tradicionales (arriba/abajo) sino que implica una relación de encontrarse dentro o fuera del sistema social en los diversos ámbitos de ciudadanía. Es decir que la exclusión social conlleva que los individuos no logren ejercer de manera justa su derecho a ser ciudadanos y por ende a ser dignificados en los distintos escenarios sociales.

En consecuencia, "hablar de la exclusión social es manifestar que el problema ya no es sólo de desigualdades entre los extremos de la escala social, sino también de distancia desde el centro del cuerpo social, entre el núcleo y los que se ven rechazados hacia los márgenes" (Consejo Económico y Social, 1997, p. 3). Es decir, que en la exclusión social subyacen discriminaciones y desigualdades a nivel social que tienen sus efectos en los contextos educativos.

De hecho, en el contexto educativo existe una construcción y un reconocimiento de situaciones, contextos, factores y dinámicas "que potencian los procesos de vulnerabilidad y/o riesgo de exclusión escolar. Al tratarse de una construcción social y cultural sobre una determinada realidad nos posibilita pensar en la existencia de otras medidas alternativas" (p.32) -buenas prácticas docentes u otro tipo de respuestas a situaciones concretas desde las políticas educativas- que reduzcan o contrarresten esas situaciones de riesgo durante el período de escolaridad y que puedan paliar esa situación de exclusión escolar del alumnado. Estas potencialidades están vinculadas sobre todo con el papel de la educación en la participación social, la integración laboral, la autoestima y realización personal. No da lugar a dudas que "el aporte de la educación resulta imprescindible en la orientación de las estrategias dirigidas a reducir y eliminar la exclusión" (Jimenez, M., Luego, J., y Tabernet. N., 2009, p.39).

En definitiva, deslegitimar la exclusión social en el campo de la educación conlleva a que las instituciones educativas reconozcan y visibilicen la participación social y el ejercicio de las libertades en las prácticas del aula. De manera que esto logre contrarrestar la exclusión social que se transada en muchos casos a los procesos de enseñanza, y es tarea de la institución educativa y del docente, promover e incentivar prácticas pedagógicas en el aula donde se reconozcan las libertades de los estudiantes, y con ello se desarrollen las potencialidades cognitivas y sociales de los sujetos en beneficio de una educación del reconocimiento y de la reivindicación de la dignidad.

\section{Idea 2: De la dignidad al reconocimiento}

En un contexto de exclusión social en los ambientes educativos a partir de escenarios sociales y económicos que en definitiva coaccionan la realización del estudiante en su contexto escolar en cuanto éste tiene pocas posibilidades de desarrollarse como sujeto con derechos, dado que existe una situación hegemónica que afecta que el estudiante logre emanciparse y por ello en muchos casos se siente excluido socialmente. Igualmente esto ocurre en las prácticas estudiantiles internas en la medida que los niños pueden ser excluidos en la medida que no son aceptados socialmente por la institución educativa, bien sea por su ideología política, religiosa, orientación sexual, entre otras.

El tema de la dignidad en el contexto escolar es fundamental para recuperar la autonomía y la libertad en los estudiantes. Al respecto, el autor Etxeberria (2013) sostiene que la dignidad 
humana, "se aplica a todos los seres humanos dotados de conciencia y libertad, y como tales, capaces de moralidad. Así mismo, la dignidad es una característica de la persona que se muestra intrínseca a ella e inalienable" (p. 135). Es decir que llevar una vida digna es una condición inherente a la naturaleza humana, en la cual cada uno y todos, quienes hacen parte de un contexto educativo tienen las mismas oportunidades en equidad de formase a través de condiciones de dignidad.

Al mismo tiempo, la dignidad humana no solo nos permite definir los grupos humanos discriminados y violentados en lo más básico. Sino que nos ayuda a que descubramos las principales dinámicas de la violencia, que se nos presentan como relevantes mecanismos de la negación de la dignidad. Si somos sujetos de dignidad, somos valor en sí, fin en sí, merecemos respeto. Entonces, la dignidad es fundamental en los procesos de reconocimiento en la educación puesto que prioriza la necesidad de que el sujeto- en este caso el estudiantesea reconocido en cuanto es un ser con derechos y libertades individuales que contribuyen a su realización humana, y por supuesto a su formación integral.

Sin embargo para que la dignidad humana se visibilice en el sujeto es indispensable que se consolide el reconocimiento, como relación consigo y en relación con los otros. Reconocerse implica comprender la importancia de que la persona conozca su razón de ser en la existencia así como la capacidad de asimilar que es una persona digna y por tanto merecedora de reconocimiento a nivel individual y social.

En esa dinámica, Fascioli (2009) expone tres aspectos fundamentales del reconocimiento: Primero, se relaciona con la autoconfianza, la cual está cimentada en el valor de las propias necesidades concretas. Segundo, implica que las personas de una comunidad se reconozcan como libres e iguales, trascendiendo el carácter particular y emocional del amor. Sobre esta esfera se erige nuestro autorrespeto, porque en la capacidad de reclamar derechos obtenemos una muestra objetiva y pública de que se nos reconoce como moralmente responsables. Y, tercero, "corresponde a la valoración social que merece un individuo o un grupo por la forma de su autorrealización o de su identidad particular. Así, el sujeto es reconocido de una manera cada vez más amplia en su autonomía y su identidad personal" (Fascioli, 2009, p.4). Entonces, la autoconfianza, el reconocimiento de las libertades y la valoración social constituyen los ejes del reconocimiento en la medida que se garantiza las igualdades y las libertades de los individuos y se enfrente todo tipo de exclusión social.

Por su parte, Axel Honneth, propone tres elementos esenciales para que exista el reconocimiento en los sujetos: el cuidado afectivo, la igualdad social (garantizada por la ley) y estima social (dada por la cooperación), de lo cual se evidencia que el reconocimiento tiene como fundamento a saber, el cuidado en sí del sujeto, además, el reconocer que éste tiene unos derechos y deberes en la sociedad, y que el reconocimiento implica la capacidad de que la persona sea reconocida socialmente. Por ello, educar en el reconocimiento no puede implicar sólo inscribirse en un marco regulativo, sino que implica también "poder estimar, dar un valor concreto al aporte que el otro, diferente - docente o alumno - hace a la vida de todos" (Honneth, 2014, p.11).

Es así como el reconocimiento aparece como el aspecto central de construcción de la dignidad humana, dado que favorece que el sujeto pueda reconocerse desde su autovaloración individual y al mismo tiempo que sea reconocido por el Estado en cuanto es un sujeto con derechos y deberes individuales que lo constituyen como ciudadano; y también en cuanto es 
reconocido socialmente el individuo tiene la capacidad de ser valorado socialmente a través de la estima social que se comprende desde la cooperación e integración social que se da entre las comunidades.

Finalmente, Etxeberria (2013) menciona que el reconocimiento posibilita: el reconocimiento de la dignidad; el reconocimiento de la ciudadanía; y el reconocimiento de la diferencia, de lo cual se asume que "todos tenemos que reconocernos unos a otros, mutuamente por igual, nuestra dignidad, ciudadanía y diferencia" (Etxeberria, 2013, p.202). Frente a la intolerancia, la xenofobia y el miedo a lo diferente, al otro o a la otra - exclusión social-, la alternativa es el reconocimiento solidario de pluralidad de formas de vida, que implica el reconocimiento de un ethos pluralista que respete tanto las éticas particulares como la ética cívica. Esto quiere decir que, "se trata del reconocimiento reciproco de las carencias y ventajas de las diferentes formas de vida y la reconstrucción común de las alternativas en el marco de la pluralidad" (Comins, 2009, p.182.). Este reconocimiento de los demás como sujetos con dignidad conlleva en reconocer "que otros cuentan con nosotros y que nosotros estamos en una situación de ayuda” (p.195).

Por consiguiente, reconocer la dignidad del otro implica que se reconozcan sus libertades y por ende que es un sujeto que tiene derechos y a la vez es ciudadano. Por tanto, hablar de la dignidad al reconocimiento posibilita comprender que la identidad del ser humano contiene su sentido existencial en la medida que éste logra comprender que es un ser digno, además de esto que puede reconocerse individual y socialmente y que por ende tiene valoración social. Esto lleva a repensar cómo el reconocimiento de los sujetos- en el contexto educativo, contribuye a que en la escuela se desarrollen prácticas de reconocimiento que favorezcan las condiciones de equidad entre los estudiantes y la comunidad educativa, y para este propósito se necesita a la vez de la alteridad.

\section{Idea3: Alteridad y posibilidad del encuentro}

Para que el reconocimiento de los sujetos logre tener un carácter emancipador y liberador se requiere de comprender y visibilizar el sentido de la alteridad en la construcción de las libertades individuales y colectivas que contrarrestan la exclusión social en los escenarios educativos. De ahí que la alteridad se constituye como la capacidad que posee el sujeto de salir de sí y con ello consolidar un sentido de reconocimiento del otro como alguien que es y debe ser reconocido en su dignidad y libertad, de manera que la construcción de la alteridad se da paso a una pedagogía del encuentro donde el yo se relaciona con el tú a través de una intersubjetividad dialógica que busca que los sujetos reconozcan sus libertades por medio del diálogo y la comunicación asertiva consigo y con los demás.

En ese contexto, autores como Levinas y Dussel desarrollaron sistemáticamente una filosofía de la alteridad encaminada a consolidar una praxis del otro que lograra emancipar al sujeto de su estado de opresión y esclavitud. Desde esa perspectiva, Levinas desarrolla en su pensamiento filosófico un acercamiento a la realidad de las personas desde una situación de vulnerabilidad y de opresión socia, para luego en su pensamiento visibilizar las condiciones del reconocimiento como necesarias en la construcción de la ciudadanía, desde su categoría de la alteridad. 
Para Enrique Dussel (1995) la alteridad es el saber pensar el mundo desde la exterioridad alterativa del otro, lo que tiene como consecuencia "el reconocimiento del otro como otro diferente al sí mismo, a través del encuentro cara-a-cara con el otro, el oprimido, el pobre; es decir, alguien que se escapa del poder del sujeto y que responde más bien a una experiencia y una temporalidad que no le pertenecen al sí mismo" (Dussel, 1995, p.27). Es decir que Dussel sigue el pensamiento leviniasiano y lo contextualiza al pensamiento latinoamericano, el cual se encuentra sumergido por situaciones de opresión y de desigualdad social, de exclusión social, y al respecto propone una filosofía de la alteridad, cuya finalidad es reconocer en el otro como alguien vital de mi existencia, y para eso se necesita de incorporar el encuentro como esa posibilidad de establecer relación dialógica con el excluido social, con el discriminado, con el diferente. Por esta razón, Dussel comparte el concepto de Emmanuel Levinas sobre el otro, cuando el filósofo lituano-francés plantea que el otro es lo "absolutamente otro; anhelo activo de salir de la monotonía de uno mismo para ascender locamente a la paz, al bien perfecto, a aquellos lugares y aquella dicha que nunca conocimos" (Levinas, 1995, p.76).

Así, por ejemplo, en el encuentro de dos culturas, la alteridad implica poner frente a frente dos concepciones diferentes de la vida: "Si hay voluntad de alteridad, existe la posibilidad de la integración armoniosa entre las personas, grupos o culturas" ( Dussel, 1995, p.10), y el diálogo permitiría enriquecer a ambas partes; por el contrario, si no hay alteridad, el pueblo más fuerte tratará de dominar al otro desde el punto de vista, lo cual generaría la exclusión social de las comunidades. Cuando se reconoce la diversidad del otro se logra comprender no solo las distintas concepciones de vida sino también establecer cómo desde la diversidad del otro se puede consolidar una integración de esas diferencias y por consiguiente constituir una ética del reconocimiento que conlleva la alteridad y la promoción del encuentro entre los sujetos.

En este sentido, una ética de la alteridad no parte de un sujeto autosuficiente y autónomo sino que enfatiza en la interrelación y de esta manera genera una nueva lógica que no está subyugada por la razón de ningún sujeto que se maquine dominador, excluyente y amenazado. Igualmente, una ética de la alteridad enfatiza en la necesidad del cuidado del otro, tal como lo sugiere Gilligan (1994), o de responsabilidad hacia el otro, como lo expresa Levinas (1995), ya que en el encuentro " nace un compromiso ético más allá de la autonomía del sujeto, siendo el otro no solamente aquel ser concreto que está frente a mí, sino también todas aquellas personas que de una $u$ otra manera puedan verse afectadas por las acciones del yo" ( Levinas, 1995, p.91).

Esto posibilita entender que una ética de la alteridad en el campo de la educación se requiere en la medida que la comunidad educativa - los estudiantes, docentes y administrativos-, logren desarrollar la importancia del cuidado y la responsabilidad del otro, esto contribuye a legitimar unas prácticas educativas solidarias y corresponsables, desde las cuales la institución educativa logre comprometerse con una ética del reconocimiento donde cada uno y todos de los miembros de la comunidad educativa puedan interrelacionarse de manera afectiva, responsable y cercana a las necesidades de los demás.

En esa perspectiva, los autores Giménez y Gonzales (2013), enfatizan en que: "La cercanía del otro no es para conocerlo; por tanto, no es esta una relación cognoscitiva, sino una relación de tipo meramente ético, en el sentido que el otro me afecta y me importa, por lo 
que me exige que me encargue de él, incluso antes de que yo lo elija" (p 342). Esto genera que se desarrolle una práctica educativa donde se reconozcan las diferencias y las libertades de los individuos, y esto se consigue a través de una ética de la responsabilidad donde todos los miembros de la institución educativa asumen éticamente la necesidad de visibilizar a todos los miembros de la institución.

En efecto, se requiere establecer una relación de reconocimiento y de responsabilidad en el ser humano quien deberá despojarse de concepciones egoístas y ególatras que le impiden aproximarse a los otros desde una concepción de igualdad en cuanto a seres con la misma dignidad. Esto requiere de una reflexión profunda desde el interior del ser, que trascienda al mundo externo y haga posible el despertar de la conciencia.

Por su parte, Fisas, (2013) plantea que: "la comprensión de la alteridad emerge desde el encuentro entre lo propio y lo otro que se hace presente frente a nosotros. Las palabras clave en ese encuentro son diferencia, comprensión y reconocimiento" (Fisas, 2013, p.32). La diferencia que nos constituye como humanos y legítimos desde nuestra identidad; la comprensión desde su papel mediador en la construcción e interpretación de la alteridad como hecho social intrínseco a la heterogeneidad; y el reconocimiento legitima la presencia del otro, desde la valoración social.

Dada la riqueza de la diversidad cultural y de las relaciones de cercanía y amistad que pueden tejerse para aprender, darse, recibir y reconstruir sentidos de vida con el otro y para el otro, en un contexto de respeto. Una educación de la alteridad busca promover los intercambios de saberes y experiencias con diferentes grupos culturales en un proceso dialéctico de constante transformación, interacción y diálogo- a través del encuentro-. En ese sentido, se esto contribuye al "reconocimiento de aprender del que es diferente a cada persona y de la riqueza que se encuentra implícita en la misma diversidad que conforma el grupo social" (MEN, 2013, p.19). Entonces, el respeto por lo diverso permite que se consolide una educación inclusiva donde se respete al diferente por su diferencia, esto contribuye a dar un trato igual a todos como individuos, de acuerdo a su condición de dignidad, para reconocerles iguales derechos civiles, políticos y sociales, esto tiene que ver con la construcción de la ciudadanía.

En resumen, para que se consolide una ciudadanía escolar, en la cual los estudiantes desarrollen en sus prácticas de aula ejercicios de reconocimiento y de alteridad, desde los cuales se legitima la convivencia con el otro- con el compañero de clase-, a partir de comportamientos y actitudes éticas resilientes y dialógicas que van acompañadas de una ética del encuentro que posibilita que todos y cada uno de los estudiantes logren en su praxis educativa contribuir a una educación del reconocimiento y de la alteridad que responde y afronte las distintas situaciones de exclusión social que afectan la dignidad y el desarrollo integral de los adolescentes en sus procesos de enseñanza y aprendizaje en la escuela.

\section{Idea 4: Pedagogía del reconocimiento a través del aprendizaje cooperativo}

La importancia de consolidar una pedagogía del reconocimiento en los contextos educativos radica en cuanto esta pedagogía posibilita construir procesos de enseñanza resilientes, empáticos y transformadores en las prácticas educativas en el aula. Para lograr esto, se necesita que los centros educativos se conciban como espacios idóneos para el mejoramiento 
de la convivencia, la cual se da como "la interrelación dada entre los diferentes miembros de la comunidad educativa con incidencia significativa en el desarrollo ético, socio-afectivo e intelectual del alumnado" (Tuvilla, 2004, p.236), así pues, la convivencia permite el fortalecimiento de una democracia auténtica y una ciudadanía comprometida.

Por consiguiente, una pedagogía del reconocimiento en el contexto de la educación es fundamental para fortalecer los procesos de convivencia y de ciudadanía en la comunidad educativa, y para este propósito se necesita de "algunos aspectos que deben ser tenidos en el aula como son: la cooperación, el aprecio por la diversidad, la expresión positiva de las emociones y la resolución de conflictos" (p.237). Estos principios de convivencia son necesarios para legitimar en las prácticas educativas pedagogías de reconocimiento que contribuyan a visibilizar la dignidad de los sujetos y la valoración de diversas formas de expresión social de los estudiantes en sus procesos de enseñanza.

En la cooperación el estudiante tiene la capacidad de colaborar cooperativamente en el aula, desde una relación empática con sus compañeros. Mientras el aprecio por la diversidad busca legitimar y reconocer la pluralidad de formas de vida en el aula, es decir, el respeto a la diversidad ideológica, sexual, religiosa y cultural que son necesarias en procesos de equidad y de igualdad social en la escuela.

En relación a la expresión positiva de las emociones se busca que los estudiantes logren comprender cognoscitivamente y afectivamente cómo el manejo de las emociones facilita el tener relaciones positivas en cualquier grupo humano, y por consiguiente ellos pueden desarrollar relaciones solidarias, justas y cariñosas con sus compañeros de clase. Desde esta visión, se logra comprender cómo el manejo de las emociones favorece a su vez la resolución de conflictos en la escuela, en la medida que el estudiante logra reconocerse y reconocer al otro en equidad, y que por tanto es necesario resolver aquel conflicto y así restaurar la relación con el compañero, y con esto se logra mejorar la convivencia en la institución.

Además de lo anterior, para mejorar la convivencia se requiere, según Tuvilla (2004) de un buen ambiente socioeducativo, esto quiere decir la necesidad de desarrollar programas de prevención primaria y adoptar un modelo de análisis y de intervención para mejorar el clima escolar y las prácticas educativas y, los problemas sociales y de relación de los miembros de la comunidad educativa. A partir de los programas de intervención y de intervención del clima escolar se busca que la comunidad educativa desarrolle procesos de concientización de la convivencia escolar para fortalecer el clima escolar en el aula de clase, el cual se caracteriza por "lo positivo de las relaciones interpersonales, por un sistema de reglas y normas claro y coherente en su aplicación y por un nivel bajo de victimización, intimidación o maltrato" (p. 256).

Para lograr esto se requiere de: a) La formación en competencias ciudadanas y el trabajo en el fortalecimiento de actitudes prosociales para la transformación del conflicto a nivel local; y b) la consolidación de una cultura del reconocimiento a partir del respeto a la dignidad humana, orientada a la transformación de los valores y de los imaginarios propios de una cultura de la violencia y exclusión social (Salamanca, Casas, Otoya, 2008, p. 43). Es decir, que una pedagogía del reconocimiento necesita de que los estudiantes se formen en competencias y habilidades sociales que les permita reconfigurar el sentido de la equidad y de la justicia en sus procesos de enseñanza y aprendizaje; además que las prácticas educativas 
logren cimentarse en los valores y una cultura del reconocimiento que permite visibilizar las libertades y las dignidades de los sujetos en el contexto educativo.

Esto se consolida a través del aprendizaje cooperativo, el cual fomenta en los estudiantes relaciones positivas necesarias para construir la confianza, el compromiso, las actitudes prosociales y las habilidades para entender y comprender al otro (Saldarriaga, 2004). Así el fundamento del principio pedagógico de este modelo parte de la interacción social con los otros y por tanto promueve aprendizajes significativos en el aula. De esta manera, en el aprendizaje cooperativo el estudiante desarrolla su confianza para comprender la actitudes de los demás y por ende comprender al otro como interlocutor válido y digno de reconocimiento.

Para desarrollar esto se requiere de tres formas de desarrollar el Aprendizaje Cooperativo (AC); el Aprendizaje Formal que se desarrolla con grupos de estudiantes para "alcanzar algunas metas específicas. En este aprendizaje se busca que el estudiante desarrolle sus habilidades y competencias en pro de sus objetivos personales que van de la manera del trabajo cooperativo en el aula. El aprendizaje Informal el cual se aplica con grupos temporales de estudiantes para "realizar actividades cortas y los Grupos de Base Cooperativos que se emplean para actividades de larga duración en las que se necesita establecer relaciones fuertes entre sus integrantes" (Saldarriaga, 2004, P.34). En este aprendizaje se generan relaciones empáticas con diversos grupos del aula de manera que se reconozcan las diferencias entre los grupos y con ello se reconozcan las identidades que emergen de las experiencias grupales en el aula.

Para finalizar aparece el Aprendizaje Cooperativo, el cual es una estrategia que fomenta en los estudiantes actitudes de respeto y colaboración mutua, dando la posibilidad para que los niños y jóvenes comprendan la importancia de trabajar con otros para alcanzar metas comunes a pesar de las diferencias individuales. En este aprendizaje cambia el rol del docente dándole más autonomía al estudiante, para que éste pueda desarrollar relaciones armónicas en el aula y además consolidar actitudes de liderazgo y de solidaria en equipo.

Adicional a lo anterior, Tuvilla (2004) resalta la importancia del Aprendizaje a través del servicio al ser "el conjunto de prácticas pedagógicas que intentan conectar experiencias de servicio a esferas específicas del conocimiento, con el doble propósito de hacer uso de ese conocimiento y de desarrollar habilidades ciudadanas que soporten la participación activa en procesos democráticos" (Tuvilla, 2004, p. 76).

En el Aprendizaje del servicio se busca que el docente genere herramientas pedagógicas a través de la solidaridad y el servicio entre los participantes de la clase. Este aprendizaje facilita que los estudiantes desarrollen habilidades ciudadanas, en la cuales se promueve una ética cívica que pretende que cada uno de los compañeros de la clase asuman responsablemente un actitud de servicio a los demás, desde unos aprendizajes cooperativos que son necesarios para consolidar una pedagogía del reconocimiento en el aula de clases.

Por esta razón, el Aprendizaje a través del servicio permite que los estudiantes desarrollen la responsabilidad, es decir "den más importancia al bien común y se involucren con mayor voluntad en la solución de problemas de la comunidad, empleando habilidades de cooperación, comunicación, autoconfianza y empatía" (Tuvilla, 2004), en la medida que todos son responsables de actuar empáticamente y cooperativamente en beneficio de la 
equidad y de la justicia en la práctica del aula. La idea del Aprendizaje del servicio en los contextos educativos no es otra que reconocer las diferencias que existen en la comunidad educativa, y desde ahí desarrollar un aprendizaje solidario que propenda por consolidar una convivencia pacífica y por ende comunidad.

En resumen, una pedagogía del reconocimiento posibilita comprender las diversas expresiones de las prácticas sociales que se encuentran en la escuela donde el estudiante puede desarrollar un proceso de enseñanza y aprendizaje empático y cooperativo, desde el cual se afinque en éste la capacidad de reconocerse y de reconocer la diversidad de expresiones en el aula en su acercamiento a los otros, de este modo, se puede minimizar la exclusión social en las prácticas educativas.

Para lograr este cometido se requiere de incentivar el Aprendizaje del servicio en los estudiantes a través de la autoconfianza, el compromiso, las actitudes prosociales y habilidades que permiten que éste se comprenda y pueda comprender al otro, y desde ahí construir comunidad. Puesto que esto facilita que el estudiante logre generar estrategias de solución en cuanto se presenten conflictos en el aula y con ello se pueda enfrentar todo tipo de exclusión al interior del aula y por supuesto en otros escenarios sociales.

\section{Conclusión}

Del presente artículo se concluyó la importancia de deslegitimar la exclusión social en el campo de la educación dado que esto afecta las condiciones de dignidad y de reconocimiento de los sujetos, en tanto excluir conlleva apartar a alguien de un escenario social, cultural y educativo. Desde esta visión, se resaltó la necesidad de visibilizar en los contextos educativos el ejercicio de las libertades individuales a través de la participación social y de prácticas de reconocimiento en la escuela que contribuyan a legitimar precisamente las dignidades de los sujetos en cuanto son ciudadanos en la sociedad. Para lograr esto se requiere incentivar en la escuela prácticas pedagógicas, en las cuales se reconozcan: la libertad, la tolerancia, el respeto por el otro y un reconocimiento activo que favorezcan las relaciones interpersonales en la clase y por ende se construyan relaciones dialógicas que son vitales para comprender la pluralidad de formas de vida que emergen en el aula.

Entonces, es fundamental profundizar en la educación actual en prácticas del reconocimiento- desde la dignidad humana-, puesto que coadyuvan a garantizar que el estudiante sea reconocido desde su autovaloración individual y al mismo tiempo sea reconocido a nivel social. Esta autovaloración individual que acontece en lo social tiene en la alteridad la concepción de que el sujeto logre salir de sí mismo y a partir de esto construir una práctica del reconocimiento del otro como alguien que debe ser reconocido en su dignidad y libertad. Es decir, que desde una perspectiva de la alteridad se logra desarrollar en el contexto educativo prácticas liberadoras que propenden por fomentar la equidad social y la justicia social en los procesos educativos y de esta manera responder a todo tipo de exclusión social- económica, social, religiosa, cultural-, que va en contravía de los derechos de los ciudadanos y del ejercicio de la ciudadanía. La consolidación de una visión de la alteridad en las prácticas educativas presupone desarrollar una pedagogía del encuentro, desde la cual el yo se relaciona con el tú con base en una intersubjetividad dialógica en la cual a partir del diálogo, de los consensos, de los acuerdos y de la comunicación asertiva se 
desarrollan prácticas de cuidado entre los sujetos en la medida que en diálogo activo posibilita el reconocimiento de las diversidades en las prácticas del aula.

En consecuencia, propiciar el encuentro en los procesos educativos permite que se estructure una pedagogía del reconocimiento en los procesos de enseñanza, y esto se consigue a través del aprendizaje del servicio, el cual facilita que los estudiantes desarrollen competencias ciudadanas y habilidades sociales en beneficio del reconocimiento de las libertades individuales y sociales. Un aprendizaje del servicio favorece que en los contextos educativos se promuevan habilidades de cooperación, de comunicación y empatía en la comunidad educativa en beneficio de la equidad social.

\section{Referencias}

Comins, I., (2009). Filosofía del cuidar: una propuesta coeducativa para la paz. Granada: EPUB

Consejo Económico Social (1997). Informe del consejo económico social. Madrid: UNESCO Cortina, A., (2003). Él mundo de los valores. Madrid: Ediciones Nobel.

Dussel, E. (1995). Introducción a la filosofía de la Liberación. Ensayos preliminares y bibliografía. Bogotá, D. C.: Nueva América.

Etxeberria, X., (2013). La educación para la paz reconfigurada. La perspectiva de las víctimas: Madrid. Catarata

Honneth. A., (2014). El derecho a la libertad. Esbozo de una eticidad democrática. Barcelona: Paidós.

Fascioli, A., (2011). Justicia social en clave de capacidades y reconocimiento. Revsita de Filosofía, (1): 24- 49.

Fisas, V., (2007). Educar para una cultura de paz. Barcelona

Gilligan, C. (1994). La moral y la teoría. Psicología del derecho femenino. México, D. F.: Fondo de Cultura Económica

Jiménez y González, A. (2013). Colombia: un mosaico de conflictos y violencias para transformar. España: Editorial Dykinson.

Jimenez, M., Luego, J., y Tabernet. N., (2009). Exclusión social y exclusión educativa como fracasos. Conceptos y líneas para su comprensión e investigación. Revista de Currìculum y formación del profesorado, (3): 12-46.

Ministerio de Educación Nacional, MEN, (2013). Lineamientos - Política de Educación Superior Inclusiva.

Navarro, O. (2008). El «rostro» del otro: Una lectura de la ética de la alteridad de Emmanuel Levinas. Contrastes. Revista Internacional de Filosofía, (XIII), pp. 177-194.

Salamanca, R., Casas, A., y Otoya, A., (2009). Educación para la paz. Experiencias y metodologías en colegios de Bogotá. Bogotá: PUJ. Salamanca, R., Casas, A., y Otoya, A., (2009). Educación para la paz. Experiencias y metodologías en colegios de Bogotá. Bogotá: PUJ.

Saldarriaga, A., (2004). Aprendizaje cooperativo. Bogotá: Uniandes

Tuvilla, J., 8 2004). Convivencia escolar y resolución de conflictos. Andalucía: Gredos. 